\title{
The Spatial-Temporal Characteristics of Urban Construction Land Expansion in Wuhan, Central China
}

\author{
Qianwen $\mathrm{Han}^{1}$, Yan $\mathrm{Yu}^{1, \mathrm{a}}$, Shanshan $\mathrm{Hu}^{2}$, Yue Chen ${ }^{1}$, Yuanyuan $\mathrm{Ke}^{1}$ and Yan Tong ${ }^{1}$ \\ ${ }^{1}$ Department of Regional Planning and Management, School of Resources and Environmental Engineering, Wuhan University of \\ Technology, 430070, China \\ ${ }^{2}$ Guangzhou Water Supply Company, No.12, Zhongshanyi Road, Yuexiu District, Guangzhou, 510030, China
}

\begin{abstract}
Data of construction land in Wuhan city were obtained from remote sensing image in different periods. Based on the spatial analysis function of GIS, the characteristics of construction land expansion were identified by several methods to analyse the spatial-temporal features of Wuhan city area from 1995 to 2015, which included expansion speed, expansion elasticity, contribution rate of expansion, centre-ofgravity shift, and quadrant orientation. The results showed that the construction land area increased gradually from 1995 to 2015 in Wuhan city, and the expansion speed first increased and then decreased. The direction of construction land expansion in Wuhan city was expanded to the southwest significantly. The construction land expansion can't meet the needs of population growth. Jiangxia district contributed most to the expansion of Wuhan city.
\end{abstract}

\section{Introduction}

With the accelerating process of urbanization, the expansion of urban construction land (UCL) has become more and more difficult to control. Therefore, studying the spatial and temporal characteristics of UCL expansion and clarifying the development of UCL are crucial for guiding urban development planning and protecting limited cultivated land resources and ecological environment. At present, numerous studies have been conducted on urban expansion control, urban expansion mode and influence of urban land expansion [1-3], in which GIS and RS technologies was combined to quantify, simulate and predict urban expansion. Previous literature on urban expansion has yielded substantial achievements. Liu and Wang [4, 5] had defined the mode of urban expansion by using relevant methods. Liu and Li et al. [6-9] had simulated urban expansion based on the landscape ecology theory. Pan et al. [10] had examined the characteristics of urban expansion by applying the relevant theory. In general, $3 \mathrm{~S}$ has been widely used in various studies of urban construction land expansion. In particular, the use of multi-temporal remote sensing data has greatly enriched the depth of urban expansion and provided data support. Most of existing studies mainly focused on the influence factors of the expansionary driving force. However, there are few of studies on the characteristics of spatialtemporal such as expansion rate, expansion intensity and development direction of urban construction land. Under such a circumstance, we have explored the development law of construction land since 1995 in Wuhan city, which could provide a reference for policymakers to achieve sustainable development in Wuhan city.

\section{Study area and data}

Wuhan city, located in the eastern of the Jianghan Plain, is the capital city of Hubei Province in China. There are 13 districts in Wuhan city: Jiang'an, Jianghan, Qiaokou, Hanyang, Wuchang, Hongshan, Qingshan, Dongxihu, Caidian, Jiangxia, Huangpi, Xinzhou and Hannan.

We collected Landsat TM / ETM + multispectral remote sensing data from 1995 to 2015 every five year. The remote sensing data came from geospatial data cloud (http://www.gscloud.cn). Remote sensing data needs to be processed to obtain land use classification maps. First, in ENVI5.1, the original RS data was preprocessed by geometric correction, mosaic, cropping and band fusion, and then the images were supervised and classified by using maximum likelihood classification. Types of land use include cultivated land, woodland, water bodies, construction land and unused land. Accuracy tests showed that the classification Kappa coefficients are more than $85 \%$, which has met the analysis requirements. Second, based on GIS technology, we extracted the data about the change of construction land use in Wuhan city in five periods, which provide data support for analysis of the spatial-temporal characteristics of the urban expansion. The extraction results of construction land are shown in Figure 1.

*Corresponding author: ${ }^{\text {a } y y h r o s e @ w h u t . e d u . c n ~}$ 

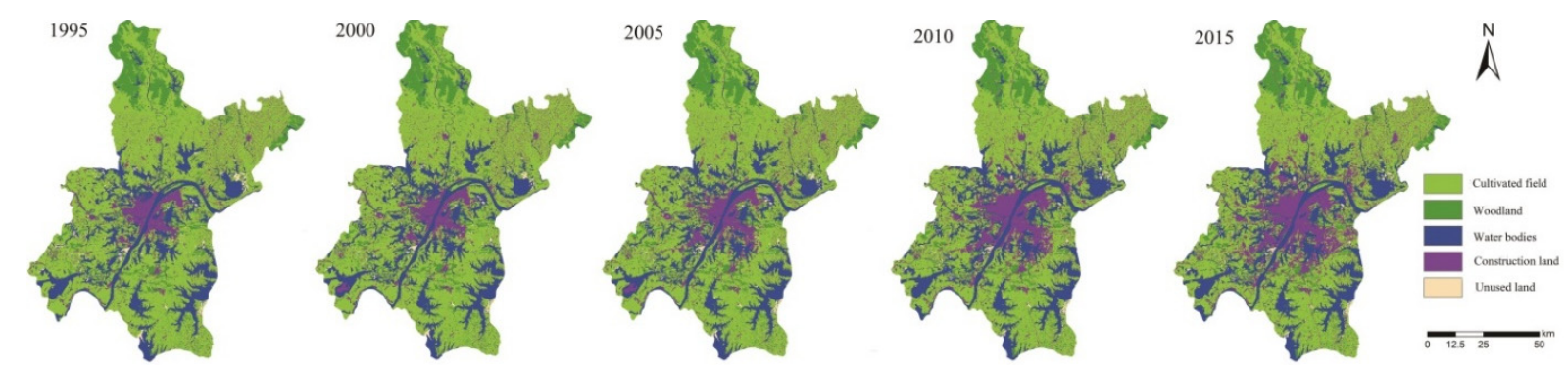

Figure 1. Distribution map of urban land use in Wuhan city from 1995 to 2015.

\section{Spatial-temporal characteristics of urban construction land expansion in Wuhan}

The methods of expansion speed, expansion contribution rate, centre-of-gravity shift, quadrant orientation and contribution rate were selected for analysing spatial-temporal characteristics of UCL expansion in Wuhan. We comprehensively analysed the spatial-temporal changes of UCL expansion during 1995-2015, and then revealed the spatial and temporal evolution of urban expansion.

\subsection{Temporal variation of urban construction land expansion}

Temporal variation of UCL expansion refers to the variation of urban land use in time during the study period. In this paper, the development characteristics in Wuhan city during the period of 1995-2015 were analysed by expansion speed and expansion elasticity.

\subsubsection{Expansion speed of urban construction land}

Expansion speed of UCL expresses the change of UCL area per unit time. It could be expressed as formula (1).

$$
V=\frac{U_{b}-U_{a}}{T}
$$

Where, $V$ is the average annual expansion rate. $U_{a}$ and $U_{b}$ are the area of UCL for the initial year and the end of the study period, respectively. $T$ is the period.

The expansion speed of UCL was divided as Highspeed (10 sq.km per year), Rapid (6-10 sq.km per year), Moderate (2-6 sq.km per year), Low-speed (2 sq.km per year). Since 1995, the construction land in Wuhan city expanded by nearly $40 \%$. Table 1 described that UCL expansion in Wuhan city has different speeds in different period. Urban development was relatively rapid during 1995-2010, 19.67 sq.km per year, 23.78 sq.km per year and 14.23 sq.km per year in the three periods respectively, all of which belonged to high- speed expansion. Meanwhile, "Wuhan City Circle" and "the Rise of Central China" were put forward. Subsequently, Wuhan city was approved by the China State Council as a central city in Central China. Wuhan " $1+8$ " had formed a network of "1 hour traffic". The increasing economic exchanges with the surrounding cities also promoted Wuhan's development. Since 2010, the development of urban construction land is a rapid expansion. Obviously, Wuhan city has paid more attention to coordination and sustainable development. In order to alleviate the harm caused by the blind expansion of the city, the expansion of the city must be controlled as much as possible.

\subsubsection{Expansion elasticity of urban construction land}

Expansion elasticity of UCL was measured the expansion characteristics in Wuhan city.

$$
R_{i}=\frac{A_{i}}{P_{P o p}}
$$

Where, $R_{i}$ is expansion elasticity of the UCL in the $i$-th period, $A_{i}$ and $P o p_{i}$ are the average growth rate of the UCL urban population in the $i$-th period, respectively.

As described in Table 1, the expansion elasticity of UCL decreased year by year from 1995 to 2005, but it still was higher than the standard value of 1.12 . This indicated that the expansion of UCL in Wuhan city was faster than the population growth. After 2005, the expansion elasticity of UCL rapidly dropped below the standard value, indicating the continuous development of urban economy and expansion of UCL can't meet the UCL caused by population growth. The uncoordinated relationship between population growth and the expansion of UCL will caused forests and arable land to be occupied so that eventually bring about a series of environmental problems. Therefore, it is necessary to develop land resources rationally so as to promote coordinated and sustainable urban development. 
Table 1. Expansion speed and elasticity of urban construction land from 1995 to 2015 in Wuhan.

\begin{tabular}{|c|c|c|c|c|c|c|}
\hline Years $\quad$ Index & Area $\left(\mathbf{k m}^{2}\right)$ & $\mathrm{V}\left(\mathrm{km}^{2} /\right.$ year $)$ & Types & $A_{i}$ & Pop $_{i}$ & $\boldsymbol{R}_{i}$ \\
\hline $1995-2000$ & 98.35 & 19.67 & High-speed & 0.1876 & 0.0552 & 3.3988 \\
\hline $2000-2005$ & 118.90 & 23.78 & High-speed & 0.1909 & 0.0696 & 2.7423 \\
\hline $2005-2010$ & 71.16 & 14.23 & High-speed & 0.0960 & 0.2211 & 0.4340 \\
\hline $2010-2015$ & 45.08 & 9.02 & Rapid & 0.0555 & 0.0840 & 0.6601 \\
\hline
\end{tabular}

\subsection{Spatial variation of urban construction land expansion}

\subsubsection{Centre-of-gravity shift}

The centre-of-gravity of UCL will also change a certain amount during the process of urban development, which reflects the shift of urban planning priorities. Centre-of-gravity coordinates and the centre-of-gravity shift can be calculated by:

$X=\frac{\sum_{i=1}^{n}\left(A_{i} \times x_{i}\right)}{\sum_{i=1}^{n} A_{i}}, \quad Y=\frac{\sum_{i=1}^{n}\left(A_{i} \times y_{j}\right)}{\sum_{i=1}^{n} A_{i}}, d=\sqrt{\left(X_{1}-X_{2}\right)^{2}+\left(Y_{1}-Y_{2}\right)^{2}}$

Where, $X$ and $Y$ represent the centre-of-gravity coordinates of UCL; $\mathrm{A}_{\mathrm{i}}$ represents the area of the $i$-th patch; $x_{i}$ and $y_{i}$ are coordinates of the $i$-th patch. $\mathrm{d}$ represents the shift distance; $X_{1}$ and $Y_{1}$ are the coordinates of centre-of-gravity; and $X_{2}$ and $Y_{2}$ are that of the reference year.

The centre-of-gravity of UCL in Wuhan has been migrating to the southwest with $6516.51 \mathrm{~m}$ (Figure 2). During 1995-2000, the expansion was shifting to the northwest with $1839.71 \mathrm{~m}$. In 2000-2005, the centre-ofgravity migrated to southwest with $1487.90 \mathrm{~m}$. During 2005-2010, the centre-of-gravity shifted to southwest with $145.51 \mathrm{~m}$. During 2010-2015, it expanded to southwest with $3043.39 \mathrm{~m}$. In the past five years, there is a more obvious UCL expansion except the East Lake.

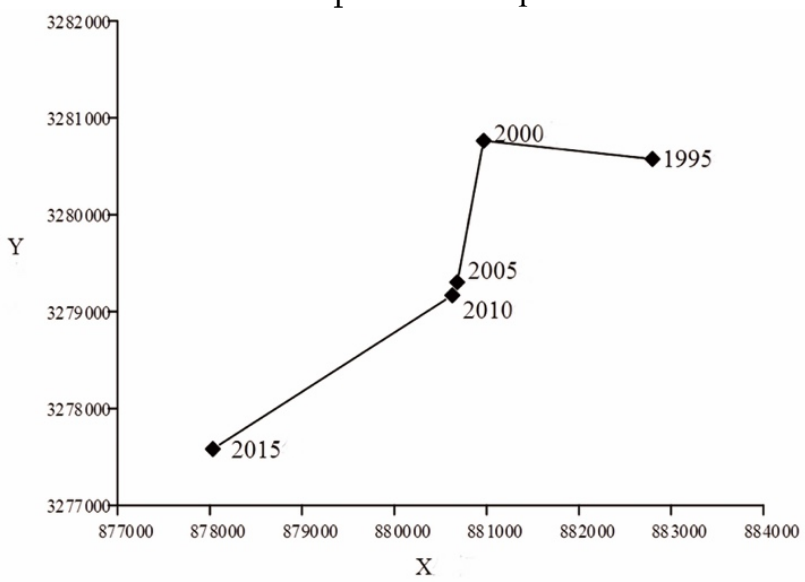

Figure 2. The centre-of-gravity shift.

\subsubsection{Quadrant orientation}

The expansion direction was divided into eight quadrants. The expansion rate of construction land was calculated and the spatial evolution of the construction land expansion in Wuhan city was also made with different time frames (Figure 3). Wuhan city expanded in all directions in four time periods, but the expansion rates were obviously different. During the period from 1995 to 2000 , it expanded mainly to the southwest and southeast with the expansion rates of 27.07 sq.km per year and 21.56 sq.km per year, respectively, and the northeast direction was only 2.10 sq.km per year. The overall expansion was the fastest from 2000 to 2005 , with the expansion rate reaching 32.99 sq.km per year in the southwest. During the period from 2005 to 2010, the expansion concentrated in the west and south directions, and the expansion rates in other directions were all below 10 sq.km per year, at a slow-expansion. The expansion from 2010 to 2015 occurred in the west, followed by the north, while in the other directions, the expansion was slow. This showed in 1995-2015, the west in Wuhan city was in a state of rapid-expansion. The southwestern direction was rapidly expanding until 2005, with a slow development after 2005 and a slow expansion. The northwest, north and northeast areas was at a slow-expansion. Therefore, the differentiation of Wuhan construction land expansion was very obvious.

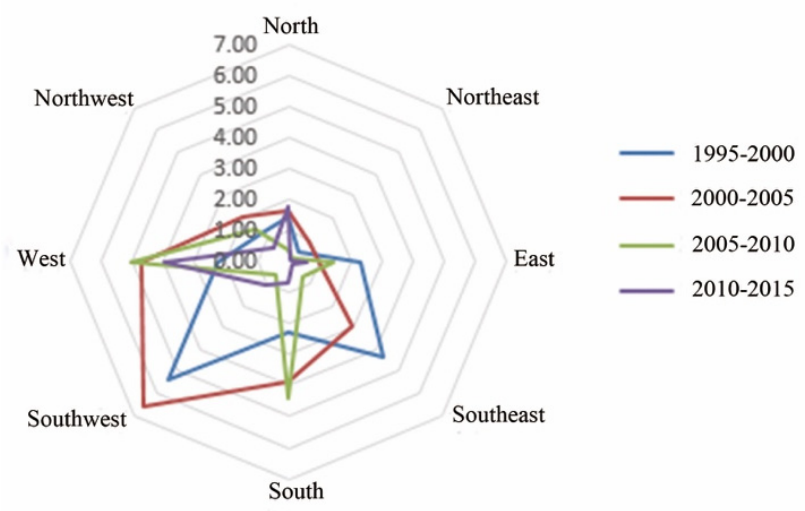

Figure 3. The Radar chart of rate of urban construction land expansion.

\subsubsection{Contribution rate of each administrative region expansion}

After studying the direction and trend of expansion, we discussed the expansion of UCL in various administrative districts. According to the difference of the contribution rate of the 13 administrative districts in Wuhan city, we analyzed the differences in the expansion of each district during the period from 1995 to 2015, in which the contribution rate of UCL expansion is:

$$
E C R=\frac{B_{(i, t 2)}-B_{(i, t l)}}{B_{t 2}-B_{t l}} \times 100 \% .
$$


Where, ECR represents the contribution rate of expansion, $B_{(i, t j)}$ indicates the UCL area of administrative area $i$ at time $t j$, and $B_{t j}$ depicts the UCL area of the entire area at time $t j$.

According to Figure 4, during 1995-2000, the differences in the contribution rate of each region's expansion were not obvious. The contribution of Xinzhou district reached $11.12 \%$, which was at the highest level. During the period of 2000-2005, the contribution rate of expansion in Jiangxia district and Hongshan district changed obviously. Among them, the contribution rate of expansion in Jiangxia district was the largest, while the change in other areas was not obvious. From 2005 to 2010, the contribution rate of expansion in Huangpi district increased the biggest, with nearly 4 times as much as that in the previous time period. In addition, Jiangxia district and Huangpi district, where the contribution rate of expansion was at a high level, accounted for $28.38 \%$ of the total contribution rate in Jiangxia district, which is the maximum value of the entire study period. From 2010 to 2015, due to the slowdown of expansion of construction land during this period, the expansion contribution rate of most regions was reduced, in which the expansion contribution rate of Jiangxia Region was the most obvious. Overall, during the study period, the expansion of Jiangxia district had the greatest impact on the overall expansion of Wuhan city. The reason is that the construction land in the downtown area of Wuhan city was developed to the border in recent years, while the traffic in the northwest of Jiangxia district was very convenient. It is nearer to the Third Ring Road in the downtown area. Most of Optics Valley planning area is in Jiangxia district, and Jiangxia set up a number of development zones, such as the Miaoshan Development Zone, Hilong Island Development Zone, Bridge district and so on.

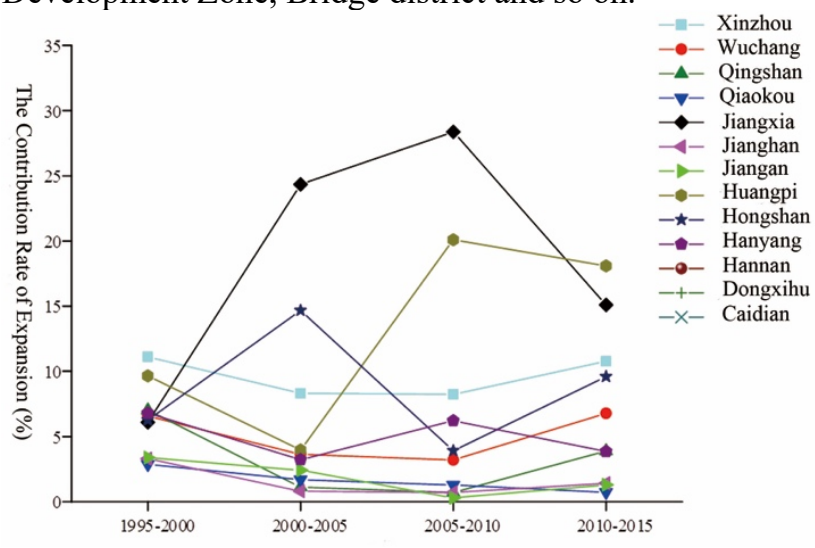

Figure 4. The contribution of each district's urban construction land expansion.

\section{Conclusions}

From 1995 to 2015, construction land in Wuhan city expanded by nearly $40 \%$, especially in the period of rapid expansion from 1995 to 2010. After 2010, the expansion began to stabilize, and the rate of expansion first increased and then decreased. In the period of rapid economic development, the pace of expansion of urban construction land obviously accelerated as same as most other cities. However, with the continuous development of the city, it is also necessary to avoid the dangers caused by the disorderly spread of cities. Therefore, the development of cities should make rational and effective use of the existing space in cities and strictly control the size of cities.

The expansion rate of construction land was higher than the population growth. This showed that people's living environment was constantly improved. After 2005, the expansion of construction land will hardly meet the demand for construction land due to population growth. As a result, the expansion of construction land caused forests and arable land to be ocuppied, which will eventually bring about a series of environmental problems. Therefore, the land must be rationally developed to promote the coordinated and sustainable development of cities.

Through the analysis of the spatial characteristics of Wuhan's expansion, we found that the main orientation of Wuhan's expansion was in the southwest, and Jiangxia district contributed most to the overall expansion of Wuhan city. The construction land in Wuhan city expanded continuously since 1995, and the economic, population and traffic had a greater guiding effect on the expansion of construction land. In the case of more and more saturated central urban area, policymakers should pay attention to the development of surrounding suburbs and the construction of transportation facilities, and make full use of the attractiveness of economic centers such as Optics Valley to promote the reseanable expansion of construction land in Wuhan city.

\section{Acknowledgments}

This work was supported by the National Natural Science Foundation of China (41471339, 41571514) and the Fundamental Research Funds for the Central Universities of China (185208017).

\section{References}

1. E.H. Wilson, J.D. Hurd, D.L. Civco. Remote Sens Environ 86, 275-285 (2003)

2. C. Sun, Z. Wu, Z. Lv, et al. Int. J. Appl. Earth Obs. 21, 409-417(2013)

3. C. Xu, M. Liu, C. Zhang, et al. Landscape Ecol. 22, 925-937(2007)

4. J. Liu, X. Wang, D. Zhuang. Acta. Geogr. Sin. 58, 885-892( 2003)

5. J. Gao, J. Chen. Geogr Res 33, 1892-1907 (2014) 
6. X. Liu, X. Li, Y. Chen, et al. Acta. Geogr. Sin. 64, 1430-1438 (2009)

7. P. Wu, D. Zhou, H. Gong. Acta. Ecologica Sinica. 32, 4270-4277(2012)

8. J. Zhang, T. Ouyang, Z. Zhu, et al. Ecol. Environ. 19, 410-414 (2010)

9. Q. Liu, et al. J. Arid. Land Resour. Environ. 27, 4752(2013)

10. J. Pan, W. Han. J. Nat. Resour. 28, 470-480 (2013) 